\title{
Banksy y Damien Hirst - Estrategias artísticas frente a la sociedad de consumo
}

\author{
Ulrich BLANCHÉ \\ Ruprecht-Karls-Universität, Heidelberg
}

Artículo recibido: 29-3-2012 / Aceptado: 8-6-2012

REsumen. ¿Cuál es el posicionamiento de Damien Hirst y Banksy frente a la cultura del consumo? ¿Qué estrategias adopta cada uno de ellos para distanciarse del ciclo del consumismo? ¿Cómo representan el capitalismo en su obra? Grosso modo: Hirst exagera la radical secularización de nuestra sociedad y Banksy, acercándose a la caricatura, critica decisiones de tipo político mediante la exhibición de las contradicciones inherentes a ellas.

Palabras clave: Street Art consumption, consumerism consumer culture, graffiti, Banksy, Damien Hirst, YBA, British art.

ABSTRACT. What is Damien Hirst's and Banksy's attitude towards consumer culture? What strategies choose the artists to get out of the consumption cycle? In what way do these phenomena of capitalism appear in their art? Generally speaking, Hirst exaggerates the radical secularization of our society; Banksy is politicizing similar to the cartoon by uncovering society's inherent contradictions.

Key words: Street Art consumption, consumerism consumer culture, graffiti, Banksy, Damien Hirst, YBA, British art.

Zusammenfassung. Wie gehen Damien Hirst und Banksy mit Konsumkultur um? Welche Strategien wählen die Künstler, um aus dem Konsumkreis zu entfliehen? Auf welche Weise tauchen diese Phänomene in beider Kunst auf? Diesen Aufsatz zusammenfassend überspitzt Hirst die radikale Säkularisierung unserer Gesellschaft; Banksy politisiert ähnlich der Karikatur durch sein Aufdecken von inhärenten Widersprüchen.

Stichworte: Street Art, Konsum, Konsumgesellschaft, Graffiti, Banksy, Damien Hirst, YBA, Young British Art, 1990er Jahre.

Banksys inhaltliche Bezüge sind fast ausschließlich kritischer Natur, es wird Konsumkritik, Gesellschafts- und Systemkritik geübt - konkret am traditionellen Kunstmarkt sowie am Kapitalismus generell, wobei insbesondere der aus Banksys Sicht oft zu unkritische, unbewusste Umgang des Betrachters mit Kunst (jedoch auch mit dem sich in Kunst reflektierendem Leben) Ziel seiner Kritik wird.

Auf einem oberflächlichen, zitierendem, fast parasitären Level beschäftigt sich Damien Hirst mit allgemeinen, zeitlosen Themen wie Tod, Religion, Liebe oder Wert(-en) - abstrakten Vorstellungen, die unabhängig vom tagespolitischen soziokul- 
turellen Hintergrund sind, beziehungsweise „irgendwie immer" passen. Damit ist, wie bei multinationalen und globalen Marken seine Produktpräsentation universell, überall gleich anwendbar ${ }^{1}$. Zugleich bleibt Hirsts Kunst damit oft Allgemeinplatz, vage und oberflächlich und spiegelt damit die Konsumkultur. Was Ullrich über Konsumprodukte sagt, lässt sich auf Hirsts wie teilweise auf Banksys Kunst übertragen:

„Wo aber geht das, was Produkte bieten, über geläufige Assoziationsketten hinaus? [...] Daß es den meisten Herstellern genügt, Projektionsflächen zu schaffen, auf denen sich möglichst viele verschiedene Konsumenten wiederfinden mögen, sollte als Antwort auf diese Frage [...] bereits genügen: Die meisten Narrative verenden nach der zweiten und dritten Assoziation. “2 . Banksys Werke sind dagegen überspitzt, moralisch wertend, wie Karikaturen, und damit oft von der Kenntnis des gerade politisch-sozial Aktuellen abhängig. Zentrale Bildmotive seiner Kunst waren etwa vor 20 Jahren noch nicht existent und sind unter Umständen in 20 Jahren nicht mehr verständlich, wie auch seine Kunst größtenteils ephemer ist. Bei Hirst verhält es sich genau umgekehrt. Beide Künstler gehen bei ihrem Umgang mit demselben Phänomen, das Bolz beschreibt, ins jeweils andere Extrem: „Je moderner, das heißt differenzierter, arbeitsteiliger und damit unübersichtlicher die Gesellschaft wird, desto größer die Sehnsucht nach Einheit und Ganzheit[...]", zumindest für den einzelnen Menschen oder Käufer/Betrachter:

„Statt eingebettet in eine selbstbewusste, generationenüberdauernde Familie oder Sippe zu leben und als Teil eines großen Ganzen [...] zu fungieren, ist er [der Mensch, Anm. UB] selbst ein - freilich nur kleines Ganzes. [...] Unabhängigkeit und Selbstbestimmung bedeuten also nicht zuletzt eine

\footnotetext{
${ }^{1}$ N. KLEIN, no logo, London 2000, p. 116.

${ }^{2}$ W. ULLRICH, Habenwollen, Frankfurt/Main, 2006,
}

Minderung der Chance, über die eigene biologische Lebensdauer hinaus zu existieren“”.

Diese Lücke versucht die Konsumkultur zu schließen, wobei sie sich bei bewährten Ganzheitsstiftern bedient. Während Banksy statt dem Ganzen einzelne konkrete Phänomene und Situationen des beschriebenen Konsumkultur-Wirrwars behandelt, für die er eine aktive Lösung als möglich aufzeigt, spiegelt Hirst ironisch dem Betrachter analog zur Konsumkultur Einheit und verlässliche Ganzheit in seiner Kunst vor, wobei er insbesondere auf die Bildsprache und den eingeschriebenen Wert anderer Bereiche, etwa Religion und Medizin zurückgreift, wie dies wechselwirkend auch Werbung macht:

„Modernes Design und Marketing haben es, unterstützt von zahlreichen Wissenschaften, dazu gebracht, daß man Dingen heute dieselben Fähigkeiten nachsagt wie seit zweihundert Jahren den Werken der Kunst: Sie bahnen Zugänge zu Erinnerungen, fiktionalisieren die Alltagswelt, transformieren Identitäten, eröffnen Zukunftsperspektiven“4. Hirst geht den umgekehrten Weg und wendet die Errungenschaften von Public Relations, Werbung, Marketing, Design, Psychologie, Soziologie oder Konsumforschung dazu an, mechanisch perfekt scheinende Kunstwerke zu erschaffen.

Im Gegensatz zu Banksy - und dies gilt für Hirsts Werk generell - führt dieser augenscheinlich Konsumphänomene nur vor, ohne Partei zu ergreifen. Das heißt, er gibt vor, nur zu betonen, dass die gezeigten Phänomene "sehens-wert" sind, jedoch nicht, ob das Gesehene als gut oder schlecht zu bewerten sei. Damit macht sich Hirst gleichzeitig zum Beobachter, Kritiker und Verfechter unserer Konsumvorstellungen; durch sein symbolisches wie betont reißerisches Präsentieren von Konsumprodukten zeigt er, dass in einer Konsumgesellschaft ,[z]wischen Symbolischem und Spektaku-

\footnotetext{
${ }^{3}$ Idem, s, 196-197.

${ }^{4}$ Idem, s. 193
} 
lärem [...] keine Unterscheidung möglich[... ]"ist ${ }^{5}$.

Während Hirst nicht nur Kunstwerke, sondern zugleich ironische Luxusartikel und Statussymbole schafft, betont Banksy auf seine Art das gespaltene Verhältnis von Kunst und Ware: Obwohl er Klischees oft im doppelten Sinne des Wortes schablonenhaft darstellt, geht er doch oft über gängige Visualisierungen von einseitigen Phrasen der klassischen linken Konsumkritik hinaus und zeigt ironisch bis zynisch Zusammenhänge etwa zwischen Konsum und Krieg, Nationalismus, Umweltverschmutzung, Unterdrükkung, Ausnutzung - zwischen Konsum und Macht auf. Beide Künstler machen über die Einseitigkeit und das Klischeehafte der üblichen konsumkritischen Plattitüden lustig - auch über die eigenen.

Damien Hirst thematisiert immer das Verhältnis des Menschen zu Dingen, wie er sich als Künstler durch den Besitz und Konsum von Dingen definiert. Er muss den Menschen nicht (oder selten) abbilden, da dieser sich selbst, seine Hoffungen, Wünsche und Ängste in den Hirst'schen Dingen, Kunstwerken/Konsumprodukten teils wörtlich widerspiegelt: „Oft ergibt sich die Frage, wer der Konsument und wer das Produkt ist - oder sind die beiden Welten bereits schon lange eine?"“6 Der zeitgenössische Mensch wurde in einer Konsumgesellschaft selbst zum Kunst- oder Konsumobjekt, zur self branded Ich-AG, nach dem Tod wird mit den Machenschaften Gunter von Hagens gelegentlich selbst der menschliche Leib zum (wenn auch wissenschaftlich verbrämten) Konsumprodukt.

"So übernehmen Menschen Strategien der Markenhersteller für sich und bieten sich wechselseitig als Projektionsflächen dar, die jenen die Erfüllung eben der Wünsche in Aussicht stellen, die gerade am lebhaftesten

${ }^{5}$ J. BAUDRILLARD, Kool Killer oder der Aufstand der Zeichen, Berlin, 1978, s. 10.

${ }^{6}$ M. HOLLEIN, C. GRUNENBERG, Shopping. 100 Jahre Kunst und Konsum, Ostfildern-Ruit, 2002, s. 14. sind. Sie werden dadurch Konsumprodukte und Konsumenten zugleich, wobei sie als letztere zugleich immer auf ein besseres Angebot warten[...] $]^{\mu 7}$.

Sich als Mensch, seine Wünsche und Vorstellungen sieht der Betrachter in Hirsts Kunst reflektiert. Aufgrund des Todes der alten Ideologien blieb in der westlichen Welt nur der Konsumismus als Sinnstifter übrig:

,[...] Außenhalt bieten heute weder die Religion noch die Nation, weder die Familie noch die revolutionäre Idee. Das macht die Entstehung des Konsumismus als Ersatzreligion verständlich “8. Ganz ein Kind der Postmoderne, ist Damien Hirst nicht mehr nur Beobachter und Seismograph seiner Zeit, die den „Tod des Autors“ wie das „Ende der Ideologien“ sah, sondern er wird mit-,,schuldig" - „[i]n einem System ohne Sinn gibt es keine Unschuld mehr ${ }^{\prime \prime 9}$ - indem er akribisch ganz entgegen dem "l'art pour l'art"-Prinzip potenzielle Konsumentenwünsche erfüllt, die letzteres wie seine Kritik zumindest vordergründig mit einschließen.

Sowohl Hirsts Konsumparadies-Illusion wie Banksys politisch-aktivistischer Ansatz drohen, in der gegenwärtigen postmodernen Konsumgesellschaft eines mit allen Werbe- und Verkaufsstrategien gewaschenen Käufers/Betrachters als mechanischer Kitsch oder "Gutmenschentum" abgetan zu werden. Dem wirken beide Künstler durch unterschiedliche Arten von Abmilderung oder Stabilisierung durch den vielbemühten „Bruch“ entgegen, der häufig mit Ironie, (De-) Platzierung, Überidentifizierung oder einem „kleinen Schock“ erreicht werden kann.

${ }^{7}$ E. ILLOUZ, in Die Tageszeitung, 26. April 2004. p. 13, zitiert nach W. ULLRICH 2006, s. 55.

${ }^{8}$ N. BOLZ, Das Konsumistische Manifest, München, 2002, s. 98.

${ }^{9}$ J. BAUDRILLARD, 1978, s. 11. 


\section{DER KONTROLLIERTE „SCHOCK“}

Einerseits instrumentalisieren Hirst sowie Banksy klassische Kitschmotive und appellieren an „,das Gefühl“, das alte Bedürfnis nach heiler Welt, ungetrübtem Optimismus und Süße, das optisch befriedigt wird. Das Wissen andererseits, es sich handele sich etwa um echte tote Tiere bei Hirst und bei Banksy die Tatsache, dass seine Ruinenromantik vergleichbare Street Art immer im Bewusstsein konsumiert wird, dass er das jeweilige Motiv illegal und unter abenteuerlichen Umständen anbrachte, verleiht beider Werk Authentizität mittels kleiner, kontrollierter "Schocks". Realität kommt bei beiden ins Spiel, die Assoziationen verlagern sich jedoch vom „lieblichen“ Märchenbuch und der Kinderillustration zu ernsthaften Themen, Leben und Tod. Genau besehen kann man den kontrollierten Schock, den Hirsts wie Banksys Kunst auslösen mag, jedoch auch als eine der Gegenwart angepasste, zeitgenössisch-postmoderne Version von Kitsch sehen. Beider Kunst bedient Kitsch und gibt zugleich vor, das Gegenteil zu sein. Letztendlich will doch beides gefallen, um leichter konsumiert werden zu können. Damit versuchen beide Künstler in ihren Werken ein gängiges Problem der Konsumkultur zu lösen: „Comfort gained, pleasure lost $^{\prime \prime 10}$. Auch Konsumprodukte sollen immer zugleich aufregend und beruhigend sein, denn ", $[w]$ as nicht neu genug ist, ist langweilig - was zu neu ist, ist befremdlich“11. Hirst wie Banksy spiegeln damit die Konsumgesellschaft und die rein konsumorientierte "Schwester" der Kunst, die Werbung, etwa provokative Anti-Werbung und Schock-Strategien wider.

Banksys eigentlich klassisch narrativillustrative Motive brauchen immer einen ungewöhnlichen, ungekünstelten realistischen Hintergrund, sein Herausstellungsmerkmal, das er auch versucht - teilweise

${ }^{10}$ N. BOLZ, Das Konsumistische Manifest, München, 2002, s. 89.

${ }^{11}$ Ibídem. erfolgreich - auf seine Werke in Innenräumen zu übertragen. Doch dort zeigt sich auch der Unterschied zwischen beiden Ansätzen: Hirsts Tiere sind tot, wie seine Kunst dinglich, verkäuflich, konsumierbar ist. Der Großteil von Banksys Kunst ist ephemer und damit nicht (ver-)käuflich, zugleich optimistischer als Hirsts Kunst, da sterblich und lebendiger. Das materielle, verkäufliche, potenziell ewige Element ist Hirst letztendlich wichtiger als Banksy, bei dem eher die nur ausgeführte dokumentierte Idee wichtig ist oder die Tatsache, dass diese Teile real sein müssen, um die von ihm intendierte Aussage und Authentizität zu haben.

\section{(IM-)MATERIELLES}

Die künstlich/künstlerisch mit tatsächlichem materiellem Mehrwert in Form wertvoller Materialien aufgeladene Kunst Damien Hirsts reflektiert die Flucht des zeitgenössischen Menschen in materielle Werte, die als "letzte Bastion“ von Glaubwürdigkeit, Authentizität und Wert gesehen werden oder letztere ersetzen kann. Dies sind Konstrukte, mit denen besonders in der postmodernen Gegenwart (jedoch auch schon etwa in der Kunst Duchamps oder Warhols) gespielt wurde/wird, deren Wichtigkeit in Kunst und Leben seither stetig zunimmt und die die durch das „Ende der Ideologien“ geschlagene Lücke zu schließen versuchen.

Nicht nur aufgrund ihrer Seltenheit haben Gold und Diamanten eine besonders lange Geschichte als Träger von Wert, einen Glaubwürdigkeits $\neg$ vorsprung, den Hirst für seine Zwecke in seinen Werken instrumentalisiert: Er versucht damit zu illustrieren, dass (seine) Kunst nicht nur in gleichem Maße, sondern mehr Glaubwürdigkeit verdient hat, da die materiellen Werte ja nur den Glanz der sinnstiftenden Möglichkeiten von Kunst verstärken.

Um tatsächlich herauszufinden, ob Hirst wertvolle Materialien verwendete, müsste man seine scheinbar kostbaren, reliquiengleichen Kunstwerke zerstören und 
einer genaueren Untersuchung unterziehen. Seine Werke handeln also zum Einen von der zeichenhaften Aussage des jeweiligen Stoffes, zum Anderen vom Vertrauen in „, das Authentische".

„Dabei wird deutlich, dass die Auseinandersetzung mit der Materialität der Kunst die spezifische institutionelle Rahmung zu manchem Paradoxon führt. Teilweise geschützt durch das Glas der Vitrine, immer jedoch mit einer Warnung versehen. Die kategorische Anweisung lautet, die Objekte nicht $\mathrm{zu}$ berühren. Wir müssen der Aussage der Beschriftung glauben. Material wird hier nur geschaut, nicht betastet. Wir dürfen seine Wärme nicht erfühlen, seine Schwere nicht überprüfen. Das Authentizitätsversprechen der Materialität ist in der Kunst nicht nachprüfbar ${ }^{\prime \prime 12}$. Hirst wie Banksy verwenden Geld als Ausdrucksmittel in ihrer Kunst, Banksy ganz konkret, Hirst in erster Linie indirekt. Die Kunst Hirsts bewegt sich zwischen Kunst und Geld, vergleichbar etwa mit Schmuck; Hirsts Kunst wie das Konsumprodukt Schmuck bestehen aus wertvollen Materialien, die finanziellen und ideellen Mehrwert kombinieren:

„In anderen Fällen ist es gerade ein hoher Preis, der einem Produkt mehr Verheißungspotential verleiht. Ihn deuten Kunden als Beleg für Potenz, die in einem Artikel steckt. Im Extremfall - etwa bei Schmuck oder Kunst - erscheint die Ware dann sogar als Symbol des Geldes oder, noch bemerkenswerter, als dessen Veredelung “13.

Derartige Produkte, zu denen auch Hirsts Kunst zählt, „überbieten, in einer Mischung aus Gebrauchs- und Fiktionswert, noch den Wert der Phantasien, die das Geld auslöst" ${ }^{\prime 14}$. Die Frage, worin man sein Vertrauen legt, läuft heute eher darauf hinaus, wem man was im doppelten Sinne

\footnotetext{
${ }^{12}$ P. SCHNEEMANN, Physis und Thesis. Vom Wert der Kunst in der Gegenwart. In: Kodikas/ Codes. Ars Semiotica. Vol. 25. Nr. 3-4. Tübingen 2002, s. 278.

${ }^{13}$ W. ULLRICH, Op. cit, s. 64.

${ }^{14}$ Ibídem.
}

"abkauft" („,to buy into something"). Damit wird die Rolle des Geldes in einer Konsumgesellschaft bewusst gemacht, eine Rolle, die sich von ihrer ursprünglichen fundamental unterscheidet:

„Sobald die dringlicheren Bedürfnisse befriedigt sind, ist Geld nicht mehr nur Zahlungsmittel, sondern genauso ein Stimulans wie ein aufgeladenes Markenprodukt. Ein Markenartikel muß dann sogar erst eigens so entwickelt werden, daß er die Konkurrenz gegen den Top-Joker Geld bestehen kann “15.

Genau dies versucht Hirst künstlich/ künstlerisch $\mathrm{zu}$ generieren. In Kombination mit religiösen Versatzstücken in seiner Kunst weist er zudem auf die enge Verknüpfung von Geld und Religion hin, was Bolz mit Benjamin folgendermaßen ausdrückt:

„Konsequent deutet Benjamin die Banknoten als Heiligenbilder der kapitalistischen Religion. An ihnen wird konkret faßbar, was es heißt, daß die Embleme des 17. Jahrhunderts im 19. Jahrhundert als Waren wiederkehren. Banknoten stellen als reiner Ausdruck des Tauschwertes zugleich Allegorien dar ${ }^{\prime \prime 16}$.

Wurde der Kapitalismus nach Weber zunächst in Religion integriert und nach Benjamin schließlich durch diese ersetzt, droht Hirst dasselbe mit seiner ambivalenten Integration von Geld in seine Kunst. Ersteres hat dadurch Letztere nicht nur herausfordert, sondern in den Augen vieler bereits ersetzt:

„Bedrohlich für die Religion ist der universale Symbolismus des Geldes nicht als dramatische diabolische Versuchung des Mammon, des goldenen Kalbs (denn der Teufel ist der dialektische Gegenspieler Gottes; er fördert also den Glauben), sondern gerade umgekehrt „,in its quiet, rational way as a substitute ${ }^{\mu_{117}}$.

\footnotetext{
${ }^{15}$ Ibídem.

${ }^{16}$ N. BOLZ, Op. cit., s. 66.

${ }^{17}$ Idem, s. 71-72.
} 
Banksy lässt sich nicht auf einen Wettstreit von Geld und Kunst ein, vielmehr karikiert er konkret Geld, beziehungsweise das, worin es sich manifestiert, Banknoten, als in erster Linie „bedrucktes Papier", an das quasi religiös geglaubt wird; seine ephemere Kunst verschwindet (im Normalfall) wieder auf natürlichem Wege. Neben der konsumkritischen Zeichenhaftigkeit ihrer Anbringung ist das Ephemere, das Immaterielle von Banksys Kunst, seiner mahnenden Kommentare auf (und zur) Zeit seine kritische Hauptaussage zum Konsum. Zwar fotografisch dokumentierbar jedoch nicht ewig (und damit käuflich) greifbar ist Banksys Kunst analog zum menschlichen Leben zu sehen.

Banksy versucht eher „unwürdige” und betont wertlose Materialien zu verwenden, beziehungsweise für seine Street Art zumindest möglichst wenig Materialkosten aufzuwenden. Als konsumkritisches Statement soll dies verdeutlichen, dass Kunst nicht teuer produziert sein muss oder darf, um ganzheitlich und nicht im Widerspruch zur konsumkritischen Aussage „im Bildteil“ $\mathrm{zu}$ wirken. Auch der junge Hirst begann teils mit Arte-Povera-Materialien, die sich im Laufe seines Werdegangs zu wertvollen Materialien hin verlagerten - bei gleichbleibender Betonung des jeweiligen Materials. Hirsts teils tierische oder menschliche Materialien werden vom Betrachter auch motivisch aufgefasst und Banksys Motive materialistisch, sie sind collagiertes Bildmaterial, Hirsts Bilder hingegen collagierte Material„Bilder".

In den auf (schnelle und mehrmalige) Reproduktion ausgelegten Mitteln und in der letztendlichen Optik von Banksys Street Art sowie der Kunstfabrik Hirsts hallt die für die Konsumkultur typische Massenproduktion wider. Diese wird zum Einen aus Gründen der Praktikabilität und Effizienz zitiert, besitzt zum Anderen Hinweis-charakter: Gezeigt wird das Paradoxon zwischen Produkt und Kunstwerk, zwischen Kunst, die industriell aussehen und zugleich einzigartig sein will. Dieser Zustand spiegelt die
Rolle der zeitgenössischen Kunst zwischen Hochglanz-Designer-Konsumobjekt und dem von der Hand eines Künstlers geschaffenem, überzeitlichen Unikat wider.

\section{MEDIENKONSUM}

Damien Hirst und Banksy wurden nicht in erster Linie mit Hilfe der großen, traditionellen Kunstinstitutionen bekannt und „wichtig", sondern durch die Massenmedien, Hirst mehr durch Printmedien und TV, Banksy in erster Linie durch das Internet. Beider auf mediale Reproduktion ausgelegte Kunst springt in den Medien unmittelbar ins Auge, sie „funktioniert“ im Schaufenster und ebenso medial vermittelt in Wort und Fotografie, ist also visuell leicht konsumierbar, weil sie Medienregeln und -gesetze instrumentalisiert und reflektiert.

So bildet Banksy bekannte, ikonenhafte Bilder des Menschen ab, welche dem Betrachter aufgrund seines (für unsere Zeit typischen) hohen Medienkonsums bekannt sind, ob er (der Betrachter) will oder nicht. Ziel ist es, eben das tausendfach Vermittelte und Konsumierte dieser Bilder sichtbar $\mathrm{zu}$ machen und die gängige Art des aus seiner Sicht passiven, unreflektierten und unbeantworteten Medienkonsums zu kritisieren, karikieren, ironisch zu brechen oder zumindest in Frage zu stellen. Zugleich bedient er sich seiner jedoch auch auf positive Weise, um seine Botschaft zu vermitteln, erweist diesen Bildern Referenz und bestärkt mit seiner Kunst bewusst oder unbewusst die Bekanntheit von gewissen visuellen Klischees, indem er „noch eins drauf setzt.“

Medienkonsumkritik kann sich auch in ihr Gegenteil verkehren. Banksy ist sich bewusst, wie schnell er als "linker" Konsumkritiker durch alles verzeihende Prominenz zu den erfolgreichsten Vertretern des Konsums werden kann, was wiederum einem bei Ullrich beschriebenen Prinzip der Konsumkultur entspricht: „Es gibt [...] keinen aufmerksameren Verstärker linker und alternativer Lebensgefühle als die heu- 
tige Marktwirtschaft" ${ }^{\text {"18. }}$. Unbequeme Köpfe, oft eben linker, konsumkritischer Denkart sorgten laut Ullrich indirekt dafür, dass der Kapitalismus sie als Vorbild nimmt, um bestimmte Produkte erst zu entwickeln, etwa solche, die paradoxerweise Konsumkultur per se kritisieren - wie im Falle Banksys, dessen (auf Konsumprodukte) gedruckte Konsumkritik reißenden Absatz findet. Dies wiederum macht Banksy in seiner Kunst sichtbar, er entlarvt den Konsumkritiker auch sich selbst - als teils willigen und unbewussten Konsumenten oder Produzenten von Anti-Konsumprodukten.

Hirst will auf "profane“ Themen wie Medienkonsum in seinem Werk verzichten und sich stattdessen den großen Themen des Lebens, Tod, Glaube, Liebe, Hoffnung widmen. Er macht dies jedoch in der ihm (und dem zeitgenössischen Käufer/Betrachter) geläufigen visuellen Sprache, die eben eine von Werbung und Konsumkultur enorm geprägte ist. Der stets beflissen nach Überzeitlichkeit strebende Hirst verzichtet auf explizite Medienkonsumkritik, implizit wird sein Medienkonsum (wie der des Betrachters) auf formaler Ebene vielfach mittels formaler Anspielungen bedient, wiederum jedoch nur in Form einer Feststellung von der quantitativen wie qualitativen Dominanz unseres Medienkonsums. Darin kann man als Betrachter Kritik am Konsum sehen, jedoch in der ursprünglichen Bedeutung des Wortes Kritik, wo neben für und wider zunächst eine eher neutrale Bestandsaufnahme kommt. Hirst ist stolz auf seinen vielfältigen Konsum von Medien- und Popkultur und fordert mit dessen auch als Glorifizierung zu verstehenden Betonung den Betrachter auf, es ihm gleich zu tun und zu konsumieren.

\section{ÜBERIDENTIFIKATION UND WERBUNG}

Hirsts Werke, akribisch von Assistenten ausgeführt und zum Konsumprodukt getrimmt, wirken nicht nur auf Fotos im Wesentlichen sofort erfassbar (wie dies bei

${ }^{18}$ W. ULLRICH, Op. cit, s. 135.
Banksy in erster Linie oft der Fall ist), sondern sind auch in der persönlichen Nahansicht bis ins kleinste Detail makellos und perfekt. Sie kommunizieren in erster Linie mit dem Galeriebesucher, der das Werk "im Original" erwerben soll und in zweiter Instanz mit dem Betrachter in Medien veröffentlichter Bilder der Werke. Banksy hingegen, dessen Kunst in gleicher Weise oder sogar mehr durch Fotografien (in Büchern oder im Internet) rezipiert werden, sieht die Drucke und Gemälde, die seine Kunst wiederum reproduzieren, als "Souvenirs" Zeugen seiner Kunst und nicht als die Kunst selbst.

Er setzt der Konsum- und Mediengesellschaft die Verweigerung, das oft Ephemere entgegen. Hirst dagegen spielt das Spiel mehr als mit, er überidentifiziert sich mit dem "Totalitarismus" des Konsums, um das Superego des Kapitalismus explizit vorzuführen. Diese für Fehlinterpretationen überaus anfällige, von Žižek beschriebene Methode kann man leicht genauso als eine völlige Identifizierung mit dem Kunstmarkt und seinen Regeln verstehen.

Banksy subversiert generell (Herrschafts-)Zeichen des Konsums, wie er auch deren Orte und Sprache subversiert, um Konsum und seine Folgen als fragwürdig und gefährlich darzustellen. Er erreicht dies, indem er diese Zeichen in den dargestellten "fruchtbaren Moment" einer Geschichte einbettet, deren Anfang und Ende man im Bild assoziieren kann, als Antagonisten einer positiven Identifikationsfigur, die an Konsumzeichen entweder scheitert oder sie überwindet. Beide Formen sind moralisierende Sinnbilder oft humoristischen Inhalts, Karikaturen, die pointiert zeitgenössischen Konsum und seine Voraussetzungen und Folgen im linken Zerrspiegel porträtieren. Diese "Sekundenbild-Dramen“ ein Begriff 19, den Michael Diers auf Werbegrafiken anwendet, beschreibt deren Bild- und Textsprache, auf die sich Banksy und Hirst beziehen.

${ }^{19}$ M. DIERS, Fotografie, Film und Video. Beiträge zu einer kritischen Theorie des Bildes. Hamburg, 2006, s. 246 
Beide adaptieren explizit Stereotypen, etwa Slogans, visuelle Klischees und Strategien, aus Marketing, Werbung und Grafikdesign. Hirst feiert letztere und macht sie sich zu Eigen, setzt sie teils nur in einen anderen, ironisierenden Kontext. Seine künstlerische Ausdrucksweise ähnelt immer mehr der leicht konsumierbaren, nicht nur visuellen Schlagwortsprache der Werbung.

Mit seinen bildgewordenen Redensarten, auch seinen Schablonengrafiken, steht die Kunst des ehemaligen (Graffiti-)Writers Banksy oft zwischen den Medien Wort und Bild, die auch die wichtigsten Mittel der Werbung sind. Für ihn stellt Werbung und die konsumistischen Motive ihrer Macher ein Feindbild dar, deren Sprache er jedoch aufgrund praktischer Vorteile bei der Herstellung sowie der Bekanntheit ihrer Ausdrucksweise bei Banksys Zielpublikum, den Konsumenten, verwendet.

Durch örtliche Kontextverschiebung macht Hirst subtil darauf aufmerksam, dass auch Bereiche, die formal und inhaltlich den Anschein erregen, gerade keine Werbung zu machen, genau dies tun, etwa die kommerzielle Schwester der Medizin, die Pharmazie oder auch das Museum. Die klare, klinisch reine Oberfläche pharmazeutischer Verpakkung wirbt dezent für ein Produkt, welches damit (wie auch viele Kunstobjekte) meist verschleiert, dass es (auch) um Kommerz, nicht nur um die Gesundheit oder l'art pour l'art geht. Hirst erklärt die Einstellung des Konsumenten zu Kunst wie zu Medizin für fragwürdig, wie er sie zugleich bestärkt.

Werbung wird bei Hirst wie Banksy als "uneheliche Schwester" von Kunst formal zitiert, inhaltlich jedoch bei Hirst auf ironischer Distanz gehalten, bei Banksy gar als die rein kommerzielle Seite visueller Ausdruckskraft verteufelt. Die von Banksy geprägten Begriffe "Chequebook-Vandalism" oder „Brandalism“ für kommerzielle Werbung großer Konzerne auf der Straße macht deutlich, dass die wahren Vandalen (die normalerweise etwa in Graffiti-Sprühern gesehen werden) die kommerziell Mächtigen sind, da sie legal abgesichert den öffentlichen Raum verunstalten und sich aneignen.

\section{IRONIE ALS STRATEGIE}

Ironie verhindert bei Banksy das zu Ernste der künstlerischen Aussage, bei Hirst das zu Unernste. Sie ist bei Banksy ähnlich wie der Karikatur unübersehbar, bei Hirst zwar immer vorhanden, doch subtiler und bis zur teilweisen Unkenntlichkeit mit dem Konzept der Überidentifikation verwoben. Hirst mahnt nur dann, wenn man es denn sehen will, Banksy dagegen mahnt immer - wenngleich stärker humoristisch gemildert - bietet jedoch meist einen Ausweg aus der Konsum-Misere an, der bei Hirst letztendlich immer Schein bleibt, ein falsches Angebot an den Betrachter, das auf Dauer unerfüllbar bleibt.

Banksy macht mit ironisch-positiver Umdeutung von Anti-Konsummotiven sowie boshaft-negativer Umdeutung positiver Konsummotive spielerisch gemildert auf den Untergrund, den Abfall, die Kehrseite, die Gefahren der Konsumgesellschaft aufmerksam. Die positive Umdeutung macht aus Ungeziefer vermenschlichte Konsumrebellen, die negative schält die Doppelbödigkeit von vermeintlich neutralen Konsumsymbolen heraus.

Banksys Humor lässt sich aus seiner Nähe zum politischen Graffiti wie zu Techniken des Subvertisings erklären. Baudrillard schreibt bereits 1978 über die Nähe politischer Graffiti und deren Umdeutung (die deutsche Übersetzung spricht von ,VerWendung" (sic!)) von Werbung:

,[D]ie Ver-Wendung der Werbung durch die Graffiti nach dem Mai `68 [...] war eine Überschreitung, nicht weil sie einen anderen Inhalt, einen anderen Diskurs einsetzte, sondern weil sie Antwort gab, dort an Ort und Stelle, und die Grundregel der Nicht-Antwort aller Medien brach. Hat sie dem Code einen anderen Code entgegen $\urcorner g e b r a c h t$ ? Ich denke nicht: sie hat ganz einfach mit dem Code gebrochen. Sie ließ sich nicht als mit dem Diskurs der Werbung konkurrierender Text dechiffrieren, 
sondern wurde als Überschreitung sichtbar. In ähnlicher Weise spielt der Witz, überschreitende Ver-Wendung des Diskurses, nicht mit der augenblicklichen Dekonstruktion des herrschenden diskursiven Codes. Er läßt die Kategorien des Codes und der Botschaft sich verflüchtigen ${ }^{\prime 20}$..

Banksys Street Art wie seine von dieser ausgehende (wenn man so will) Gallery Art "ver-wendet" die urbanen Orte ihrer illegalen Anbringung immer, auch dann, wenn dies nicht explizit „im Bildteil“ thematisiert wird. Dadurch werden die Zeichen- und Machtverhältnisse gestört, von denen Baudrillard spricht, weil der Betrachter wie die „Machthaber" keine "Antwort" erwarten oder diese bestrafen. Die Machthaber sind wie erwähnt einerseits im urbanen Raum die großen Firmen, die nicht nur Werbeflächen kaufen, in der Galerie ist es andererseits der Kunstmarkt, beides sind Konsumkultur repräsentierende Institutionen, deren Motive wie Methoden Banksy - schon allein durch die Umstände der Anbringung - in Frage stellt, bricht, ad absurdum führt und der Lächerlichkeit preisgibt. Banksys humorvolles Vorführen/Adeln des Nicht-Legitimen stellt immer auch die Legitimität der Obrigkeit in Frage.

Der geschilderte Sachverhalt bestärkt den oft als eng empfundenen Terminus Street Art, da gezeigt wurde, dass dieses „Kunstprinzip des falschen Ortes" das Karikaturhafte, das Widersprüchliche, das Banksys Kunst ausmacht, aus seiner Herkunft vom Graffiti, dem reinen Akt der illegalen und damit immer auch politischen Anbringung wie des inhaltlich politischen Graffitis ableiten lässt.

\section{(DE-)PLATZIERUNG UND INSZENIE- RUNG}

"So much power of an image comes from where and when you see it"21.

Banksy

${ }^{20} \mathrm{~J}$. BAUDRILLARD, Op. cit, s. 112.

${ }^{21}$ BANKSY zitiert nach M. ROBERTSON, Let us spray. In: The List. Glasgow and Edinburgh events guide, edition 408. 1-15. March 2001, s. 24.
Während Banksys Kunst auf der Straße, also der "realen Welt", (de-)platziert ist, verfährt Hirst umgekehrt - er bringt andere Realitäten in traditionelle Kunstorte. Durch seine (De-)Platzierung von Konsumgegenständen, Requisiten oder ganzer Bühnen in einem Kunstkontext, führt Hirst, wenn man dies denn sehen will, Konsumdenken als oft unreflektiert vor: Medizin- und Wissenschaftsgläubigkeit, der Glaube an Religion oder den Kapitalismus, finanzielle Werte, Kitsch, das vermeintlich Schöne, das Sammeln - Konsum wird "frag"-würdig bei Hirst. Gleichzeitig feiert dieser das Haben(wollen) jedoch auch und zeigt, dass es (wenn auch nicht immer zu Recht und nicht für ewig) durchaus mit Sinn füllen kann.

Eine Form von Deplatzierung lässt sich auch in der pastoralen Szene sehen. Diese findet laut Stallabrass, der Ergebnisse Empsons zur Pastoral-Literatur auf Young British Art übertrug, heute im Urbanen statt:

"[T]he outlook embodied in pastoral has been turned from the rural to the urban, particularly to the landscape of the inner city. [...] Pastoral is plainly an art that is about common people but not for or by them. But insofar as it is an attitude of the rich, it involves them in a double view of the poor: both that the rich concept that they have better powers of expression that they can bring to full consciousness and representation the unconscious virtues of the poor, but at the same time $[\ldots]$ the poor may have an advantage over the rich" ${ }^{\prime 22}$.

Stallabrass sieht Damien Hirst, einen Künstler mit working class Hintergrund, als Vorreiter dieser Tendenz:

„Indeed one way to look at this renewal of pastoral in British art is to see its originary move as being to shift the site of pastoral from the countryside to the inner city; and again, Damien Hirst serves as the usher, with his metaphorical murder of the rural idyll" ${ }^{23}$.

${ }^{22}$ J. STALLABRASS, High Art Lite. The Rise and Fall of Young British Art, New York 2006, s. 250-252.

${ }^{23}$ Idem, s. 253. 
Dieses Konzept lässt sich auch auf Banksys Street Art übertragen. Banksy bringt seine Karikaturen auf der Straße an, wie er umgekehrt mit Street-Art-Motiven pastorale Ölgemälde umdeutet. Banksy, der wie erwähnt die Ruinenoptik des immer leicht heruntergekommenen urbanen Umfeldes dringend als authentifizierenden Kontrast seiner Werke benötigt, überträgt diese - nicht immer erfolgreich - selbst auf Galerie-Innenräume. Stets ist diese „VerWendung" des Raumes eine künstlerische Strategie, den Kontrast zwischen high und low culture, zwischen upper und working class, Elite und Undergroundkultur, Kunstmarkt-Kunst und Straßenkunst, kommerzieller Werbung und selbstwerbender Street Art immer zugunsten jeweils Letzterer darzustellen: „Modernism in ruins takes on a strongly Romantic and sublime character ${ }^{\prime \prime 24}$. Während Hirst eher die generelle Einstellung kommentiert, kritisiert Banksy konkret „modernism, particularly modernist architecture, by placing the forms of its ideals against the subject matter of what, in conventional wisdom, is its worst legacy"25. Banksy illustriert auf der Straße oft den selben Gedanken im Bildteil, den er durch den Akt beziehungsweise die Umstände der Anbringung des Werkes demonstriert. Durch Banksys eigenmächtige, da illegale Transformation (und deren mediengestützter Verbreitung) des eigentlich "öffentlichen Raumes", der so oft nur noch ein kommerzieller Durchgangsraum ist, erobert er diesen aktiv, mit einer antikonsumistischen Geste, stellvertretend für den passiven Konsumenten/ Passanten zurück und zeigt, dass diese Rückeroberung eines Einzelnen möglich und nötig ist. Banksy verwendet die Straße auch, um bekannt zu werden, um seine Kunst und seinen Namen zu promoten. Dies führte wiederum dazu, dass er selbst als Künstler, beziehungsweise seine antikommerziellen Werke, kommerziell werden, dass jedes, werbungskritisierende Werk Banksys zugleich Werbung für die

\footnotetext{
${ }^{24}$ Idem, s. 256.

${ }^{25}$ Ibídem.
}

Marke Banksy macht, was er selbst ironisch thematisiert.

Beide Künstler inszenieren ihre Werke bis zum einem Grad, ab dem die vorgeführte Inszenierung dominanter ist als das inszenierte Werk selbst. Damit reflektieren sie die zeitgenössische Konsumkultur, Banksys Medienstunts zeigen sich neben Werbung von öffentlichkeitswirksamen Aktionen etwa von Umweltschutz-organisationen oder Kaufhauseröffnungen beeinflusst.

Hirsts in Galerien und Kunstmuseen assoziierten Apotheken, Kirchen, Operationssäle, Naturkunde- oder Kunstmuseen sind immer auch als leichter konsumierbare Sensation, Spektakel, Läden inszeniert wie auch Galerien nie frei von kommerziellen Interessen sind. Indem er „Wert" an einen anderen Ort, einen Kunstort transferiert, führt er das Theatrale, das Geschminkte, das eigentlich doch (zumindest auch) für den Konsum Präparierte von beiden Orten vor, dem tatsächlichen wie dem assoziierten. Beiderlei Orte versuchen, sich hinter ihrer vermeintlichen Objektivität zu verstecken, werden jedoch von Hirst marktschreierisch demaskiert.

\section{DER SAMMLER ALS PERFEKTER KONSU- MENT}

Die in Variationen immer gleichen Motive Hirsts, das Serielle seiner Kunst, wie die für ihn typischen gesamtwerkimmanenten Anspielungen verweisen auf die Tätigkeit des Sammelns, das auch als MarketingStrategie zur Steigerung des Konsums verwendet wird:

„Mittlerweile wird den Konsumenten häufig suggeriert, sie seien eigentlich Sammler. Dazu genügt es schon, auf eine Verpackung »limited edition « zu schreiben und so einen Wertzuwachs in Aussicht zu stellen. [...] Dies ist eine ebenso sichere Methode, ein Habenwollen anzufachen, wie die Konkurrenzsituation einer Versteigerung $[\ldots]^{\prime 26}$. Die medienwirksam instrumentali-

\footnotetext{
${ }^{26}$ W. ULLRICH, Op. cit, s. 190.
} 
sierte Kunstauktion wie das aktive Kreieren von Serien, Sammlerstücken sind Methoden, mit denen Hirst nicht nur Verkäufe steigert, sondern auch Konsumkultur reflektiert, so dass nicht die Werke selbst, sondern deren Inszenierung zu Recht heute oft als das Künstlerische bei Hirst zählt: Das theatreale, medienwirksam vorgeführte In-Szene-Setzen eines im Grunde beliebigen Konsumproduktes oder Kunstobjektes, und es damit mit symbolischem Mehrwert ${ }^{27}$, mit laut Kant "Zweckmäßigkeit ohne Zweck" beziehungsweise mit "Bedeutsamkeit ohne Bedeutung" aufzuladen ${ }^{28}$, ist eine gängige von Werbung und Marketing übernommene Methode.

Auch hinsichtlich der veränderten Rezeption von Kunst übernimmt der Sammler eine Vorreiterstellung:

„Ein Sammler kann sich als Experte in Kunstfragen allein dadurch profilieren, daß er ein Kunstwerk kauft; er benötigt keine Argumente und Theorien, um dessen Wert nahezubringen. Die Preisangabe ersetzt die Begründung eines Geschmacks- oder Werturteils, der Konsum tritt [...] an die Stelle der Rezeption“" 29. Als Idealform des Käufer/ Betrachters steigert ein bedeutender Sammler das Ansehen und damit den Wert aller anderen Werke des gesammelten Künstlers. Letzterer schafft verlässlich für den Sammler in großem Umfang Serien, Nachschub gibt es immer, nicht das limitierende Hirst-Angebot bestimmt die Nachfrage, sondern nur der Preis, bei mehr Bedarf wird mehr produziert, da seine wie auch Banksys Bilder nicht mehr eine Idee verkörpern, sondern diese jederzeit wiederholbar illustrieren ${ }^{30}$.

\section{BRANDS NOT PRODUCTS}

Die künstlerische Idee wird in erster Linie in der Aura des Künstlers offenbar, ähnlich einer eingetragenen Handelsmarke

\footnotetext{
${ }^{27}$ Idem, s. 122.

${ }^{28}$ Idem, s. 48.

${ }^{29}$ Idem, s. 191.

${ }^{30} \mathrm{~J}$. STALLABRASS, Op. cit, s. 101.
}

wird deren Kraft durch die Signatur auf das Kunstobjekt übertragen, eingebrannt wie ein Brandzeichen (das englische Wort brand bedeutet Marke) oder, um im Bild der Marke zu bleiben, wie ein Logo:

„Der Name der Marke verspricht die Sicherheit der Qualität; er garantiert Enttäuschungsfestigkeit. Symbolisiert wird das vom Logo, das ja aufs Zunftzeichen, aufs Herkunftszeichen zu rückgeht, also wie

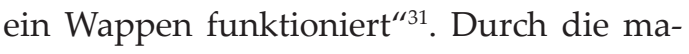
gische Berührung des Meisters (die auch ironisch gesehen werden soll/kann) wurde schon bei Duchamp der Alltagsgegenstand zur Kunst. Bolz vergleicht das Logo mit dem Begriff des Totems, den er ähnlich wie den der Ikone verwendet:

„Das Totem unterscheidet sich von Alltagsgegenständen durch seine Eigenschaft, als faszinierendes Bild zu wirken, das Gefühle an sich bindet. Im System des Konsumismus ist es das Logo, das als Totemwappen fungiert. Es können die Waren der modernen Märkte als eine Art Geheimschrift gelesen werden, in der sich unser gesellschaftliches Leben religiös chiffriert ${ }^{\text {"32 }}$.

Auch ohne Signatur oder tag ist Hirsts wie Banksys Kunst als die jeweils ihre problemlos zu erkennen, da beide ikonenhafte Bilder schufen/schaffen oder medienwirksam so verwend(et)en, dass sie zuerst mit ihnen identifiziert werden. Diese Verlagerung der Benjamin'schen Aura des Kunstwerkes auf die Persona des Künstlers resultiert aus der Tatsache, dass Hirsts wie Banksys Ideen gerade auf Reproduzierbarkeit ausgelegt sind und gar erst mit der Quantität ihrer Reproduktion zu Kunst werden. Diese Unterscheidung spiegelt ebenfalls die zeitgenössische Tendenz wider, nicht das einzelne Werk, sondern den Künstler als „Gesamtkunstwerk" zu betrachten, bei der dessen angeblich „persönliche“ Einstellung (etwa

\footnotetext{
${ }^{31}$ N. BOLZ, Op. cit, s. 121.

${ }^{32}$ Idem, s. 114.
} 
zu Kunst als Konsumprodukt), Image, Aura, Marke wichtiger und letztendlich künstlerischer ist als das einzelne Kunstwerk. Letzteres ist etwa oft Teil einer Serie und immer nur werkimmanent und in einem größeren Zusammenhang fassbar, der letztendlich nur mit der genannten „Aura des Künstlers“ zu beschreiben ist.

Beide Künstler arbeiten in Serien oder Motivserien und repräsentieren und reflektieren als etablierte Marken-Künstler in ihren Werken das für die Konsumgesellschaft ab den 1980er Jahren zunehmend wichtige Konzept des „brands not products“, welches große Konzerne begannen und das sich international durchsetzte. Dabei wird Aura, Name, Bekanntheitsgrad und Image einer Marke geschaffen und als Konsumprodukt verkauft. Unter dem Label Damien Hirst oder Banksy können dann etwa outsourced product lines (ausgelagerte Werkgruppen) fremd-,,produziert" pseudo-individuelle und pseudo-maschinelle Kunstwerke teils wie am Fließband geschaffen werden, die, teils unabhängig von der eigenen Relevanz schon allein aufgrund ihres (wie auch immer gearteten) Kontakts mit dem Star, mit der Marke Banksy oder Hirst (oder beiden, etwa durch cross marketing) "geheiligt" oder für finanziell und/oder künstlerisch wertvoll erklärt werden. Dies wird von beiden teils gefördert, teils abgelehnt und teils ironisch oder wütend reflektiert.

Die Tatsache, dass auch Banksy als Mensch im (wenn auch kritisierten/karikierten System) Kapitalismus existieren will und muss, zwingt ihn jedoch dazu, seine Kunst auch zu Geld, also zu einem Konsumprodukt zu machen, in Form von Gemälden, Drucken, Büchern et cetera. Bis zu welchem Grad diese von Banksy nach außen propagierte idealistische Einstellung tatsächlich der Realität entspricht, hängt von der Frage $\mathrm{ab}$, inwieweit man den Menschen von der Persona des Künstlers Banksy trennt; wie auch bei Hirst ist diese Frage nicht vollständig zu beantworten.
In Hirsts wie Banksys Werken wird zugleich offensichtlich, dass beide geschilderten, vom jeweiligen Künstler gewollten Ansätze an ihre Grenzen stoßen: Banksys Konsumkritik verkehrt sich teils ins Gegenteil angesichts seiner persönlichen finanziellen Erfolge, Hirsts oft als Konsum verherrlichend wahrgenommenen Werke wurden zu Wahrzeichen einer hedonistischen verschwenderischen Zeit, die den Hochmut vor dem Fall, die Finanzkrise von 2008, verkörperten und damit nicht ganz freiwillig $\mathrm{zu}$ Beispielen für Konsumkritik wurden. Ähnliches lässt sich auch über Konsumkultur sagen:

"Auch überschätzte man die Macht von Design, Marketing und Wissenschaften, wollte man unterstellen, es ließe sich vorherbestimmen, was ein Ding auslöst. Noch das ausgeklügelste »Cue-Management « läßt genügend Raum nicht nur für Flops, sondern ebenso für positive Überraschungen. Umgekehrt ist die Kunst alles andere als ein kalkülfreies Terrain, und manches, was ein Rezipient als Geheimnis oder unerschöpflich vieldeutig wahrnimmt, ist nur Folge eines geschickten Einsatzes wohlerprobter Effekte" ${ }^{\prime 3}$.

Eine Kunst wie die von Banksy und Damien Hirst, die Konsumkultur reflektiert, insbesondere auch den dazugehörigen Betrachter und die Konsumorte, instrumentalisiert/birgt immer auch die Nachteile dieser Kultur, woraus resultiert, dass ihr ähnliche Vorwürfe wie der Konsumkultur gemacht werden:

„Erst wenn Konsumprodukte eine semantische Dichte besitzen, die mit der Kunst und Hochkultur vergleichbar ist, wird die Konsumkritik ihr stärkstes Argument - den Vorwurf der Banalität, Obszönität, Oberflächlichkeit - eingebüßt haben und weitgehend verstummen “34.

\footnotetext{
${ }^{33}$ W. ULLRICH, Op. cit, s. 194-195.

${ }^{34}$ Idem, s. 199.
} 